\title{
Predicting reactivities of protein surface cysteines as part of a strategy for selective multiple labeling
}

\author{
Maik H. Jacob, Dan Amir, Vladimir Ratner, Eugene Gussakowsky and Elisha Haas
}

Faculty of Life Science, Bar-Ilan University, Ramat Gan, Israel 52900

\section{Supporting Information}

\section{Table of Content}

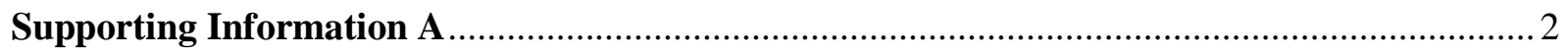

Sequence, secondary structure and single cysteine mutants of AK........................................... 2

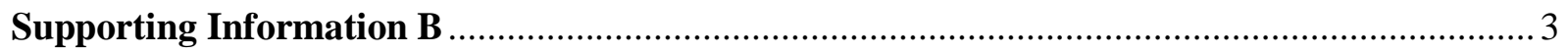

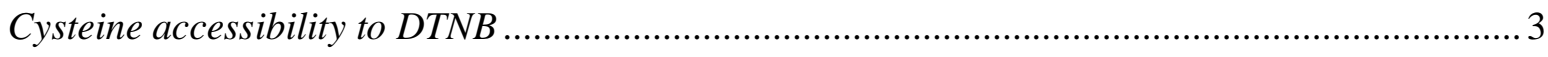

FIGURE S1: Assumed cysteine-DTNB conformation at the time of reaction........................... 3

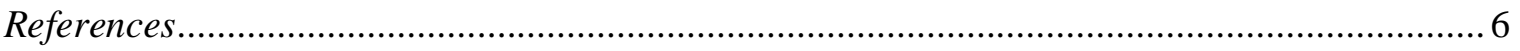

Supporting Information C .........................................................................................

Predictive power and robustness of the electrostatic models .................................................. 7

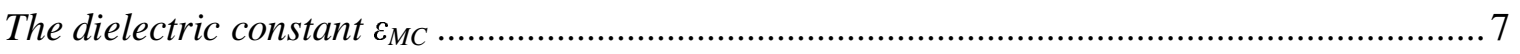

FIGURE S2: Robustness of model 1 towards changes in the model parameters...................... 8

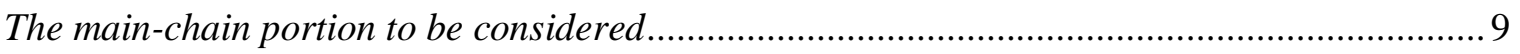

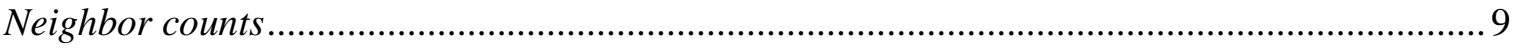

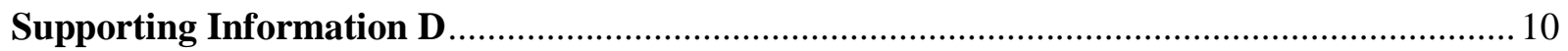

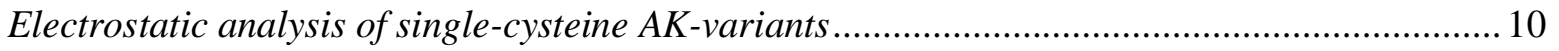




\section{Supporting Information A}

Sequence, secondary structure and single cysteine mutants of AK

Table S1 depicts the sequence and secondary structure of C77S-adenylate kinase. Residues are marked that were replaced by cysteine in the single cysteine mutants.

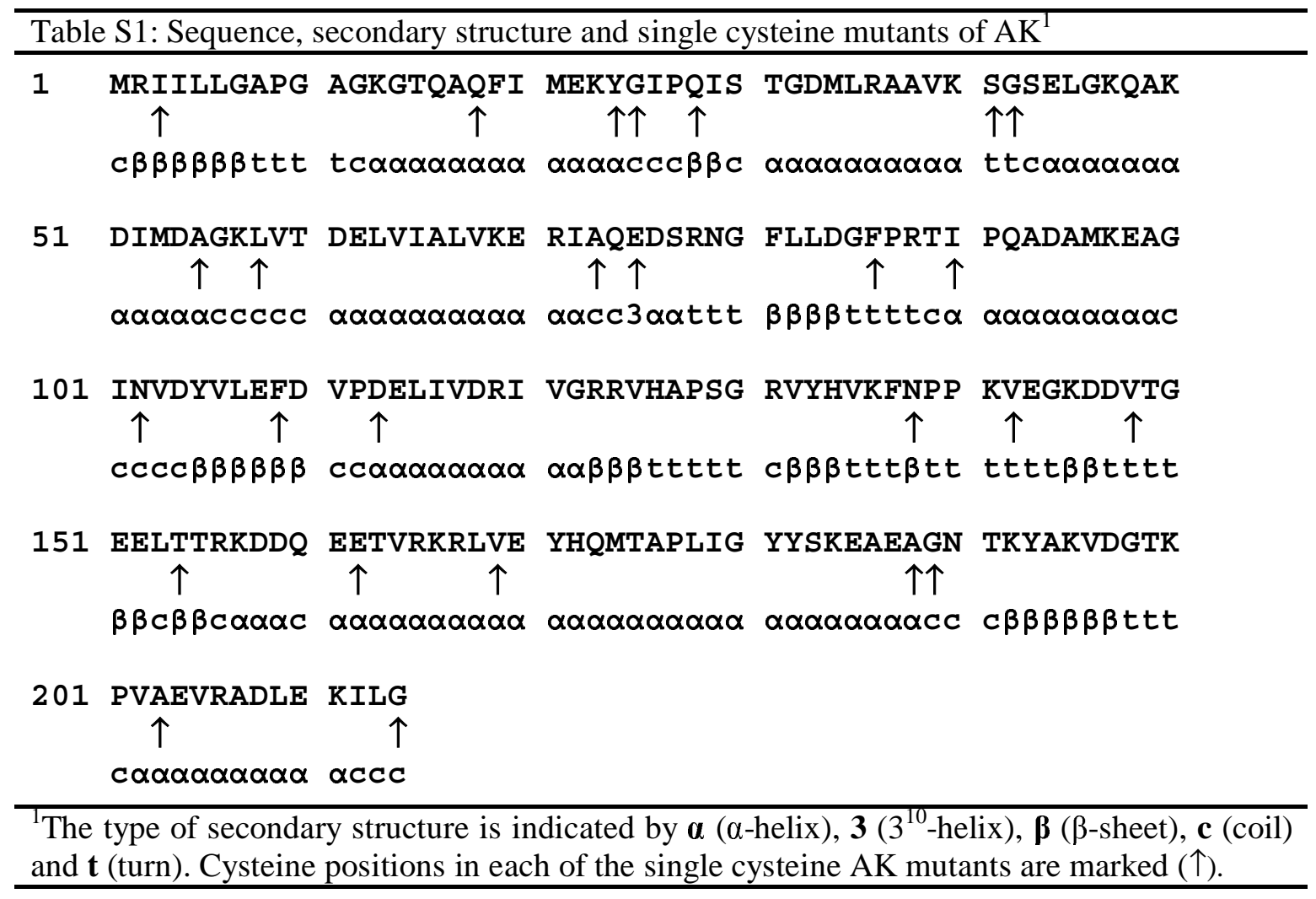




\section{Supporting Information B}

Cysteine accessibility to DTNB

Cysteine accessibility to DTNB was defined as the probability that DTNB at the time of reaction adopts a conformation that is sterically compatible with the protein surface. The extent of steric compatibility in a particular transition-state conformation was assumed to depend mainly on the kind and number of interatomic collisions.

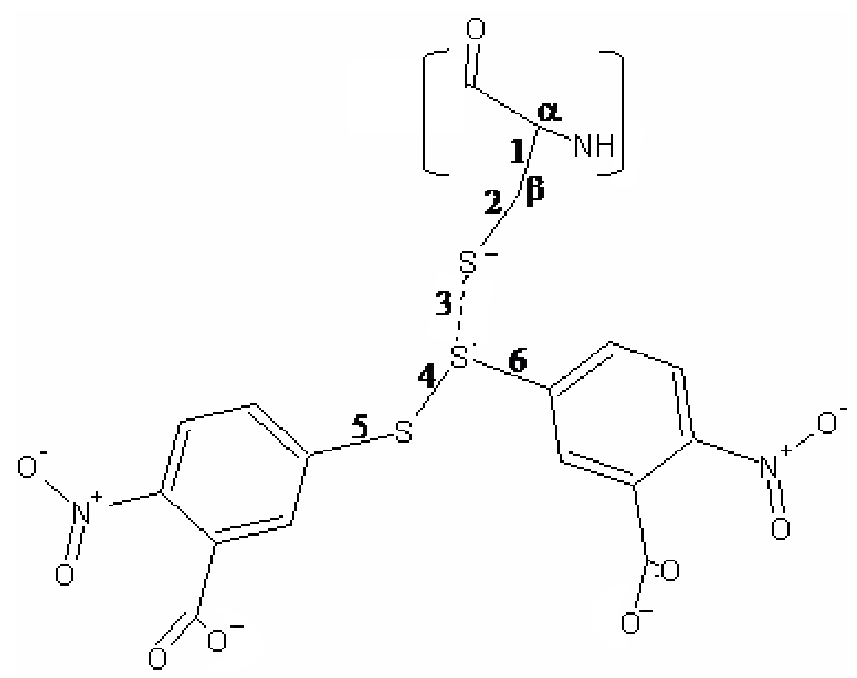

FIGURE S1: Assumed cysteine-DTNB conformation at the time of reaction.

Angles between bonds 3 and $6\left(\mathrm{~S}^{-} \cdots \mathrm{S}-\mathrm{C}\right)$ and bonds 3 and $4\left(\mathrm{~S}^{-} \cdots \mathrm{S}-\mathrm{S}\right)$ were set to $120^{\circ}$. In the simulation, 24,000 conformations were generated by rotation around the axis 1 to 6 - twelve adjustments of the dihedral angle were allowed for bond 1, five for bonds 2, 3 and 4 and four for bonds 5 and 6.

The geometry of the protein-DTNB complex, in which the reaction can occur, was modeled as described in the legend of Figure S1. Rotameric DTNB conformers were generated by stepwise rotation around bonds 1 to 6 . Twelve rotameric positions of the cysteine-sulfur atom were chosen in such a way, that the corresponding dihedral $\mathrm{N}-\mathrm{C}_{\alpha}-\mathrm{C}_{\beta}-\mathrm{S}$ angles (bond 1) were equally distributed over the sterically accessible range. Five adjustments of the dihedral-angles were allowed for bonds 2, 3 and 4 and four for bonds 5 and 6 to generate a total of 24,000 conformers. Each conformer was tested for collisions with protein main-chain and side-chain atoms. The lowest interatomic distance that corresponds to a collision was set to $4 \AA$. The coordinates of rotamers were obtained by using the atom- 
group algorithm of Zhang and Kavraki [1]. All calculations were carried out in the program

\section{Excel/VBA.}

An apparently obvious measure of cysteine accessibility is the fraction of DTNB conformations that make no clashes and are fully compatible with the protein surface. However, this fraction can be extremely small when only a single side chain is in an unfavorable orientation towards DTNB (in the crystal-structure conformation) — a reasonable measure of cysteine accessibility must take into account that a side-chain can escape from collision by rotation. Based on this consideration, we calculated compatibility probabilities for DTNB conformations. The probability, $P_{\mathrm{ICO}, \mathrm{SC}}(d, n)$, that DTNB in conformation, $d$, is sterically incompatible (ICO) with the side-chain (SC) of the neighbouring residue in sequence position $n$, is assumed to depend on the number $\operatorname{COL}_{\mathrm{SC}}(d, n)$ of collisions between DTNB and the specific side chain as expressed by equation S1.

$$
\begin{gathered}
P_{\mathrm{ICO}, \mathrm{SC}}(d, n)=1 /\left\{\exp \left[2 \cdot\left(4-\mathrm{COL}_{\mathrm{SC}}(d, n)^{1 / 2}\right)\right]+1\right\} \\
\text { for } \operatorname{COL}_{\mathrm{SC}}(d, n)>0
\end{gathered}
$$

This probability equals $50 \%$ for 16 collisions and approaches $100 \%$ for 25 collisions.

The probability that a given conformation of DTNB is incompatible with the location of a neighbouring residue should depend much less on the number of collisions between DTNB and the residue's side chain than on the number of collisions with the sterically restrained main-chain atoms of the residue (including the $\mathrm{C}_{\beta}$ atom). The probability $P_{\mathrm{ICO}, \mathrm{MC}}(d, n)$ that DTNB in conformation $d$ is incompatible with the main chain of residue $n$ should already be high for low collision numbers. Therefore, the dependence of $P_{\mathrm{ICO}, \mathrm{MC}}(d, n)$ on the number of DTNB collisions $\operatorname{COL}_{\mathrm{MC}}(d, n)$ with the main chain of residue $n$ was modeled in such a manner that $P_{\mathrm{ICO}, \mathrm{MC}}(d, n)$ already equals $50 \%$ when only four collisions are detected (eq S2). 


$$
\begin{gathered}
P_{\mathrm{ICO}, \mathrm{MC}}(d, n)=1 /\left\{\exp \left[2 \cdot\left(4-\mathrm{COL}_{\mathrm{MC}}(d, n)\right)\right]+1\right\} \\
\text { for } \operatorname{COL}_{\mathrm{MC}}(d, n)>0
\end{gathered}
$$

The probability $P_{\mathrm{ICO}}(d, n)$ that the chosen cysteine-DTNB conformer is indeed incompatible with the residue $n$ is obtained by equation $\mathrm{S} 3 \mathrm{a}, \mathrm{b}$.

$$
\begin{aligned}
& P_{\mathrm{ICO}}(d, n)=P_{\mathrm{ICO}, \mathrm{SC}}(d, n), \\
& \text { if } P_{\mathrm{ICO}, \mathrm{SC}}(d, n)>P_{\mathrm{ICO}, \mathrm{MC}}(d, n) \\
& P_{\mathrm{ICO}}(d, n)=P_{\mathrm{ICO}, \mathrm{MC}}(d, n), \\
& \text { if } \mathrm{P}_{\mathrm{ICO}, \mathrm{MC}}(d, n)>P_{\mathrm{ICO}, \mathrm{SC}}(d, n)
\end{aligned}
$$

The probability $P_{\mathrm{COM}}(d, n)$ that DTNB in conformation $\mathrm{d}$ is compatible with residue $n$ is obtained from equation $\mathrm{S} 4$.

$$
P_{\mathrm{COM}}(d, n)=1-\mathrm{P}_{\mathrm{ICO}}(d, n)
$$

The probability $P_{\mathrm{COM}}(d)$ that the probed DTNB conformation is compatible with all neighboring residues in the assumed transition state equals the product $\Pi\left(\mathrm{P}_{\mathrm{COM}}(d, n)\right)$ of the individual compatibility probabilities of all residues (S5).

$$
\begin{aligned}
P_{\mathrm{COM}}(d)= & \prod\left(P_{\mathrm{COM}}(d, n)\right) \\
& \text { for residues } n=1,2, \ldots, 214
\end{aligned}
$$


The accessibility index, $A$, of an inserted cysteine residue at each sequence position was obtained by averaging the compatibility probabilities of all 24,000 DTNB conformations (eq S6).

$A=\Sigma P_{\mathrm{COM}}(d) / 24.000$

References

1. Zhang, M., and Kavraki, L. E. (2002) A New Method for Fast and Accurate Derivation of Molecular Conformations, J. Chem. Inf. 42, 64-70. 


\section{Supporting Information C}

\section{Predictive power and robustness of the electrostatic models}

We analysed our results to determine to what extent our method can be generalized to other protein systems. To this end, we asked: (1) How strongly is the predictive power of the model dependent on the values chosen for the parameters? How robust is the model? (2) Is an appropriate choice of values important only for a distinct subgroup such as the helix-terminal residues, or for all the positions on a protein?

The Pearson linear correlation coefficient $R$ is a measure of the probability that two variables are linearly related; but it is the coefficient of determination, $R^{2}$, that reflects the extent to which the variation of the dependent variable is "explained" by the regression line, which presents a measure of the predictive power of the observed correlations. We explored how $R^{2}$ of the linear correlation between reaction rates and fractions of cysteinate, $f\left(\mathrm{C}^{-}\right)_{\mathrm{MC}, \mathrm{SC}}$, depends on the dielectric constant, $\varepsilon_{\mathrm{MC}}$, which we used to calculate the energy of cysteinate interactions with the protein main chain.

\section{The dielectric constant $\varepsilon_{M C}$}

Fractions of cysteinate $f\left(\mathrm{C}^{-}\right)_{\mathrm{MC}, \mathrm{SC}}$ were calculated via model 1 but with a dielectric constant $\varepsilon_{\mathrm{MC}}$ varying between 4 and 80 . The determination coefficients of regression equations were plotted as a function of the dielectric constant in Figure S2A. The respective slopes of the regression lines are shown in Figure S2B. Cysteines were again assigned to groups as follows: (1): residues at helix termini ( $\mathbf{\Delta}$ ); (2): other residues ( $\mathrm{O}$ ) now including C86 and C109; (3): all residues ( $\diamond)$ but excluding C3, 24 and 58 - otherwise, these three values would dominate the correlations as, here, they were calculated according to model 1, which does not account for Born penalty differences. 

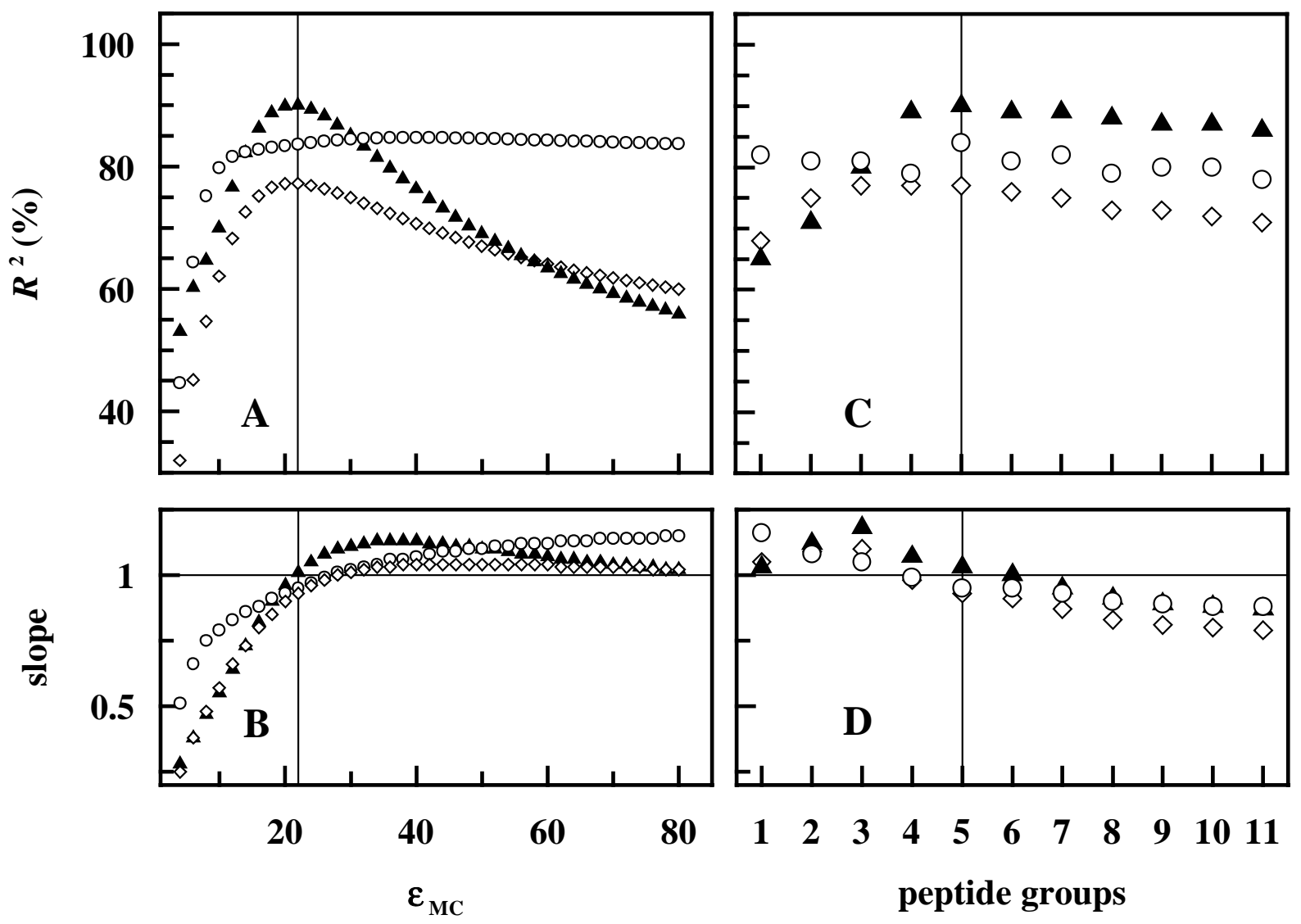

FIGURE S2: Robustness of model 1 towards changes in the model parameters.

Three different sets of cysteine residues are considered: (1) residues at helix termini $(\boldsymbol{\Delta}),(2)$ other residues ( $(\circ)$ including $\mathrm{C} 86$ and C109, (3) all residues $(\diamond)$ but excluding C3, 24 and 58.

(A) The coefficient of determination $R^{2}$ is plotted against the dielectric constant $\varepsilon_{\mathrm{MC}}$. The vertical line marks the value $\varepsilon_{\mathrm{MC}}=22$ which we used in our analysis (Table 1, Figure 2A, B). The corresponding dielectric constant $\varepsilon_{\mathrm{SC}}$ for side-chain charges was kept constant at 40 .

(B) The slope of the best linear fit of rates and fractions (-log values) is plotted against $\varepsilon_{\mathrm{MC}}$. The horizontal line indicates a slope of 1.

(C) The determination coefficient of $R^{2}$, and (D) the slope of the linear regression line is plotted against the number of peptide groups, preceding and following the respective cysteine $\mathrm{C}_{\alpha-}$ carbon in the chain, which were included in the calculation of the electrostatic interaction energy between the main chain and the cysteinyl anion. Data shown in Figures 2A, B and Table 1 were calculated by accounting for the effect of the five peptide groups flanking the cysteine $\mathrm{C}_{\alpha_{-}}$carbon on each side (vertical lines in C and D).

(D) The horizontal line indicates a slope of 1.

The determination coefficient for the helix-terminal residues $(\mathbf{\Delta})$ was highest $\left(R^{2}=93 \%\right)$ when $\varepsilon_{\mathrm{MC}}$ was set at 22 (Figure $\mathrm{S} 2 \mathrm{~A}$, vertical line). Values of $\varepsilon_{\mathrm{MC}}$ that strongly differed from 22 led to much less significant correlations as well as to regression-line slopes differing from 1. For non-terminal cysteines (०), such a strong sensitivity of the correlations towards $\varepsilon_{M C}$ was not found, when $\varepsilon_{M C}$ was varied between 10 and 80; the determination coefficient $R^{2}$ remained between $80 \%$ and $85 \%$. But regression- 
line slopes were closest to 1 when $\varepsilon_{\mathrm{MC}}$ was close to 22 . Similarly, for the group of all cysteines $(\diamond)$, the best $R^{2}(78 \%)$ was obtained when $\varepsilon_{\mathrm{MC}}$ was set at 22 . These data indicate that the electrostatic interactions between a cysteinyl anion and local main-chain dipoles are best calculated with a dielectric constant $\varepsilon_{\mathrm{MC}}$ of 22 , no matter whether helix-terminal or other cysteines are considered.

Because cysteine sites were selected based on their low rotamer scores and neighbor counts, neighboring protein atoms belonged predominantly to the same part of the protein chain as the cysteine under consideration. Only dipoles on the local protein main chain were thus included in the calculations, but the optimal portion of the adjacent main chain to be considered for predicting cysteinyl-anion formation, was yet to be established.

\section{The main-chain portion to be considered}

Fractions of cysteinate $f\left(\mathrm{C}^{-}\right)_{\mathrm{MC}, \mathrm{SC}}$ were calculated with inclusion of varying portions of the main chain as measured by the number of peptide groups flanking the $\mathrm{C}_{\alpha}$ atom of the specific cysteine along the protein chain. Both dielectric constants, $\varepsilon_{\mathrm{MC}}$ and $\varepsilon_{\mathrm{SC}}$, were held constant at 22 and 40 , respectively.

The determination coefficient of helix-terminal residues increased strongly with an increasing number of neighboring peptide groups included in the calculation (Figure S2C). When accounting for greater portions of the main chain (up to 11 peptide groups at each side of the cysteine $\mathrm{C}_{\alpha}$ carbon), the determination coefficient remained nearly constant, but the corresponding regression-line slopes became increasingly low (Figure S2D). The determination coefficient of correlations for non-terminal residues was nearly independent of the number of flanking peptide groups considered. Regression line slopes near 1 were found when three to seven peptide groups on each side were included in the analysis.

\section{Neighbor counts}

In Model 2, we used the number of atoms in a sphere with radius $r=7 \AA$ around the cysteine-sulfur atom position (neighbor count). The correlation coefficients obtained via model 2 remain almost unchanged, when the value of this radius remains in the range of 5 to $9 \AA$. 


\section{Supporting Information D}

\section{Electrostatic analysis of single-cysteine AK-variants}

In addition to Table 1, we listed in Table S1 the individual contributions of main-chain dipoles (MC), side-chain charges (SC) and the Born penalty, which was calculated from the number of neighboring atoms (NC), to the cysteine $\mathrm{p} K$. A $\mathrm{p} K$-value difference of 1 between two cysteines gives rise to an about tenfold difference in reaction rates.

Table S2: Electrostatic analysis of single-cysteine AK-variants

\begin{tabular}{|c|c|c|c|c|c|c|c|c|c|}
\hline \multicolumn{2}{|c|}{$\begin{array}{l}\text { Cysteine } \\
\text { group and } \\
\text { position }\end{array}$} & \multirow{3}{*}{$\begin{array}{c}\text { Reaction rates } \\
k\left(\mathrm{mM}^{-1} \mathrm{~s}^{-1}\right) \\
-\log k\end{array}$} & \multicolumn{5}{|c|}{$\begin{array}{l}\text { Single and combined effects of main- and side- } \\
\text { chain charges, (MC), (SC), and water exclusion } \\
\text { (NC) on cysteine deprotonation }{ }^{1}\end{array}$} & \multicolumn{2}{|c|}{$\begin{array}{c}\text { Fraction cysteinate } f^{1} \\
(\%)\end{array}$} \\
\hline & & & \multicolumn{5}{|c|}{$\Delta \mathrm{p} K$} & \multicolumn{2}{|c|}{$-\log f\left(\mathbf{C}^{-}\right)$} \\
\hline & & & MC & SC & $\mathrm{NC}$ & MC,SC & MC,SC,NC & MC,SC & MC,SC,NC \\
\hline \multirow{12}{*}{1} & 18 & -0.42 & -0.76 & 0.41 & 0.59 & -0.45 & 0.14 & 1.61 & 0.99 \\
\hline & 25 & 1.73 & 0.80 & 0.12 & 0.59 & 0.95 & 1.54 & 3.00 & 2.34 \\
\hline & 41 & -0.14 & 0.25 & -0.64 & 0.84 & -0.56 & 0.29 & 1.51 & 1.12 \\
\hline & 42 & -0.26 & 0.78 & -1.61 & 0.66 & -0.76 & -0.10 & 1.31 & 0.77 \\
\hline & 55 & 0.26 & 0.32 & -0.26 & 0.93 & 0.07 & 1.00 & 2.13 & 1.81 \\
\hline & 73 & 1.57 & 0.74 & 0.25 & 0.88 & 1.03 & 1.91 & 3.08 & 2.71 \\
\hline & 90 & 1.13 & -1.28 & 1.43 & 1.11 & 0.12 & 1.24 & 2.18 & 2.04 \\
\hline & 113 & -0.90 & -1.49 & -0.07 & 1.04 & -1.15 & -0.11 & 0.95 & 0.77 \\
\hline & 162 & -1.31 & -1.21 & -1.37 & 0.58 & -2.48 & -1.90 & 0.14 & 0.03 \\
\hline & 188 & 1.77 & 0.22 & 1.00 & 1.07 & 1.30 & 2.37 & 3.35 & 3.17 \\
\hline & 189 & 1.71 & 1.03 & 0.58 & 0.40 & 1.61 & 2.01 & 3.66 & 2.81 \\
\hline & 203 & -0.72 & -1.39 & 0.23 & 0.52 & -0.97 & -0.45 & 1.11 & 0.51 \\
\hline \multirow{10}{*}{2} & 28 & 0.88 & -0.50 & 0.60 & 1.05 & 0.06 & 1.11 & 2.12 & 1.92 \\
\hline & 58 & -1.18 & -0.38 & 0.48 & 0.34 & 0.11 & 0.44 & 2.16 & 1.27 \\
\hline & 75 & -0.62 & -0.78 & -1.63 & 0.46 & -1.74 & -1.29 & 0.48 & 0.12 \\
\hline & 102 & 1.19 & -0.34 & 0.05 & 0.73 & -0.23 & 0.50 & 1.83 & 1.32 \\
\hline & 138 & -0.72 & -0.34 & -0.99 & 0.91 & -1.57 & -0.66 & 0.61 & 0.38 \\
\hline & 142 & 0.46 & -0.10 & 0.14 & 0.94 & 0.06 & 1.00 & 2.12 & 1.81 \\
\hline & 148 & -0.16 & -1.06 & -0.92 & 0.81 & -2.13 & -1.32 & 0.26 & 0.11 \\
\hline & 154 & -0.13 & -0.41 & -0.53 & 0.82 & -1.05 & -0.24 & 1.04 & 0.67 \\
\hline & 214 & 2.17 & 0.32 & 1.01 & 0.40 & 1.47 & 1.87 & 3.52 & 2.67 \\
\hline & 169 & -0.26 & -0.44 & 0.55 & 0.87 & 0.10 & 0.98 & 2.16 & 1.78 \\
\hline \multirow{4}{*}{3} & 3 & 4.74 & 0.06 & 0.34 & 4.78 & 0.44 & 5.22 & 2.49 & 6.02 \\
\hline & 24 & 3.14 & 0.11 & -0.26 & 1.89 & 0.05 & 1.94 & 2.10 & 2.74 \\
\hline & 86 & 1.63 & -0.03 & -0.04 & 1.77 & 0.08 & 1.85 & 2.14 & 2.66 \\
\hline & 109 & 2.19 & -0.19 & 0.78 & 2.40 & 0.58 & 2.98 & 2.63 & 3.78 \\
\hline
\end{tabular}


The value $\Delta \mathrm{p} K_{\mathrm{MC}}$ represents the change of the cysteine $\mathrm{p} K$ caused by electrostatic interactions between the cysteine-sulfur atom and partial charges of the main chain, ignoring discrete charges of side chains. Similarly, $\Delta \mathrm{p} K_{\mathrm{SC}}$ accounts for the effect of discrete charges on side chains, as if main-chain charges were absent. The value $\Delta \mathrm{p} K_{\mathrm{MC}, \mathrm{SC}}$ is the change of the cysteine $\mathrm{p} K$ caused by both main- and side-chain charges (model 1, eqs 4-9). The effective interaction energy had to be calculated according to the Boltzmann distribution by averaging over all individual energies of possible cysteinyl anion rotamers (eq 5), which is why $\Delta \mathrm{p} K_{\mathrm{MC}, \mathrm{SC}}$ differs slightly from the sum of $\Delta \mathrm{p} K_{\mathrm{MC}}$ and $\Delta \mathrm{p} K_{\mathrm{SC}}$. The value $\Delta \mathrm{p} K_{\mathrm{NC}}$ is the change of the cysteine $\mathrm{p} K$ due to water exclusion. The value $\Delta \mathrm{p} K_{\mathrm{MC}, \mathrm{SC}, \mathrm{NC}}$ is the sum of $\Delta \mathrm{p} K_{\mathrm{MC}, \mathrm{SC}}$ and $\Delta \mathrm{p} K_{\mathrm{NC}}$. 